\section{Circulating miR-146a $-5 p$ in Egyptian patients with Rheumatoid Arthritis}

\author{
Raghda M. EL-Bakry', Samia M. Fadda², Rabab A. \\ Mohamed $^{3}$, Enas A. Abdelaleem ${ }^{1}$
}

${ }^{1}$ Rheumatology \& Rehabilitation Department, Faculty of Medicine, Beni-Suef University, Beni-Suef, Egypt.

${ }^{2}$ Rheumatology \& Rehabilitation Department, Faculty of Medicine, Cairo University, Cairo, Egypt.

${ }^{3}$ Clinical \& Chemical Pathology Department, Faculty of Medicine, Beni-Suef University, Beni-Suef, Egypt.
The Egyptian Journal of Immunology Volume 29 (1), 2022: 29-38. www.Ejimmunology.org
Corresponding author: Rabab A. Mohamed Department of Clinical \& Chemical Pathology, Beni-Suef University, Faculty of Medicine, Beni-Suef, Egypt.

Email: rabab.eltememy@gmail.com

\begin{abstract}
We indented to evaluate of the role miRNA-146a-5p expression level as a possible biomarker associated with the incidence of rheumatoid arthritis and the detection of its relevance with different disease parameters. The study included 60 clinically diagnosed RA patients fulfilling 2010 American College of Rheumatology / European League Against Rheumatism (ACR/EULAR) classification criteria for Rheumatoid Arthritis and 45 age and sex matched control subjects. Disease activity was assessed by Disease Activity Score 28 (DAS28). The expression levels of miR-146a in whole blood was measured using reverse transcriptase quantitative real time polymerase chain reaction (RT-PCR). There median expression level of miRNA-146a-5p was significantly higher in RA patients compared with the control group $(P=0.036)$. MiR-146a expression level was positively correlated with individual activity markers, including erythrocyte sedimentation rate (ESR) $(P=0.04)$, visual analogue scale (VAS) $(P=0.047)$ and Modified Health Assessment Questionnaire (MHAQ) $(P=0.050)$. A lower expression level of miRNA-146a-5p was detected in RA patients on antimalarial drugs as compared to patients whose treatment protocol did not include antimalarial drugs $(P=0.016)$. In conclusion, data of this study suggested a possible association between miRNA 146a-5p expression level and the incidence of RA in Egyptian patients.
\end{abstract}

Keywords: Rheumatoid arthritis (RA), MiRNA-146a, RT-PCR, DAS-28.

Date received: 23 November 2021; accepted: 23 December 2021

\section{Introduction}

Rheumatoid arthritis is a chronic autoimmune disease characterized by the presence of local and systemic inflammation where the close interaction between immune cells and soluble mediators leads to amplification and perpetuation of inflammatory and remodeling processes. ${ }^{1}$ The etiology of RA is not completely understood; however, it was suggested that it might be a result of a blend of genetic and environmental factors which ultimately ends in an over reactive immune system. ${ }^{2}$

Even though several identified genes might be implicated in the susceptibility to RA, it is still unclear how these hereditary features might result in the induction of RA. Although RA appears to be a polygenic disease, other factors 
as epigenetics might be the link to explain the different inconsistencies in the relation between genetic polymorphisms and the disease presentation. ${ }^{3}$

MicroRNAs (miRNAs) are small non-coding RNAs which have diverse roles in regulating immune functions that includes $T$ and $B$ cell selection and maturation. This implies that miRNAs might have a role in the progress of immunological tolerance and might be implicated in the occurrence of many immunological mediated disorders. ${ }^{4}$

Several studies have reported that miRNAs can be expressed in tissues, peripheral blood mononuclear cells, synovial fluid, chondrocytes and synovial cells in Rheumatoid arthritis patients. miRNAs might also be implicated in the progress, advancement and prognosis of RA. $^{5}$

MiRNA-146a was first defined by Taganov et al., 2006 who reported that the levels of expression of miRNA-146a were increased due to stimulation with lipopolysaccharides. ${ }^{6}$ However, other researchers reported that its level of expression is augmented by the action of pro-inflammatory cytokines. ${ }^{7}$ Thus, it has been linked to the pathogenesis of RA through the role of many genes associated with inflammation and apoptosis. ${ }^{8}$

As the expression of miRNA-146a is varied among RA patients and conflicting evidences exist regarding its role in disease progression, ${ }^{9}$ the current study was designed with the aim to evaluate the role miRNA-146a expression level as a possible biomarker associated to the incidence of RA and to detect its relation to disease activity and severity.

\section{Patients and Methods}

\section{Patients}

Sixty adult patients diagnosed with RA were recruited in the current case-control study. The diagnosis of RA was done in accordance with the 2010 American College of Rheumatology/European league Against Rheumatism (ACR/EULAR) classification. ${ }^{10}$ The patients were enrolled from the Rheumatology and Rehabilitation outpatient clinic and the inpatient unit, Faculty of Medicine, Beni-Suef
University from November 2019 to December 2020. Forty-five age and sex matched apparently healthy adults participated as a control group. The criteria of exclusion included patients with history of joint deformity, other autoimmune disease, pregnancy and lactation. The study protocol was reviewed and approved by the Ethical Committee of the Faculty of Medicine, Beni-Suef University of Medicine (Approval No.: FMBSUREC/03092019). An informed consent was obtained from each participant in the study.

\section{Methods}

\section{Evaluation of Disease Activity and Severity}

Patients participating in the study were subjected to complete history taking and thorough clinical examination. The patients were assessed for disability index by the modified health assessment questionnaire (MHAQ). ${ }^{11}$ The intensity of pain was assessed by a $100 \mathrm{~mm}$ visual analogue scale (VAS) $(0=$ no pain and $100=$ worst possible pain). ${ }^{12}$ The disease activity was assessed by the Disease Activity Score including a 28 joint count (DAS28ESR).$^{13}$ In all patients, plain radiograph of both hands and feet in the postro-anterior views were obtained. Van der Heijde-modified sharp score (vdHSS) was used to detect degree of radiological severity of the disease severity. ${ }^{14}$

\section{Laboratory Investigations}

Blood samples $(10 \mathrm{ml})$ were collected from the patients and the controls under complete aseptic conditions. The samples were distributed: one part $(5 \mathrm{ml})$ was used in the performance of the routine laboratory tests which included: complete blood count (CBC) (Automated Hematology Analyzer XP-300, Sysmex, Germany), Erythrocyte sedimentation rate (ESR), C-reactive Protein (CRP) (HUMATEX CRP Agglutination test, Human, Germany), Rheumatoid factor (RF) (HUMATEX RF Agglutination test, Human, Germany), Anticyclic citrullinated peptide antibodies (Anti-CCP) (Quanta lite ${ }^{\circledR}$ IgG ELISA, INOVA, USA), complete urine analysis, serum uric acid, liver function tests (AST, ALT and serum albumin) (Beckman Coulter AU480, USA) and kidney function tests (blood urea and creatinine) (Beekman Coulter 
AU480, USA). The second part of the withdrawn blood $(5 \mathrm{ml})$ was preserved in EDTA containing vacutainers and kept at $-80^{\circ} \mathrm{C}$ until used for relative quantitation of miRNA $146 a$ expression.

\section{$R T-P C R$ expression analysis of MiR-146a-5p}

RNA was sequestered from blood using miRNANeasy Mini Kit (Qiagen, Hilden Germany) according to the manufacturers protocol. Detection of miR $146 a-5 p$ expression level was accomplished in accordance with the manufacturer's protocol by real-time PCR assay using TaqMan PCR kit. The primers used for detection of miR-146a-5p and U6 expression were as follows: for miR-146a-5p, forward: TGAGAACTGAATTCCATGGGTT, reverse: GCTGTCAACGATACGCTACGTAAC-; U6, reverse: GCTGTCAACGATACGCTACGTAAC, forward: CTCGCTTCGGCAGCACA. Detection was performed using Applied Biosystems Step one TM Instrument (USA) in accordance with the manufacturer protocol. Calculation of the relative expression of miR-146a-5p was based on the threshold cycle and was normalized to U6. Data analysis has been performed by the $2^{-}$ $\Delta \Delta \mathrm{ct}$ method.

\section{Treatment}

RA patients were grouped according to the presence or absence of non-biological disease modifying anti- rheumatic drugs (DMARDs) in their treatment protocol into three groups. The treatment protocol of the first group included Methotrexate, the second group's protocol included Leflunomide, and the third group's protocol included anti-malarial drugs.

\section{Statistical methods}

The IBM SPSS ${ }^{\circledR}$ Statistics version 22 (IBM ${ }^{\circledR}$ Corp., Armonk, NY, USA) was used for analysis of statistical data. The processing of numerical data was performed using the mean and standard deviation or median and range as applicable. The frequency and percentage were used to express qualitative data. The relation between the qualitative variables was done using Pearson's Chi-square test or Fisher's exact test. Student's t-test was used for the comparison between two groups for normally distributed data while the Mann-Whitney test for the comparison of not normally distributed quantitative data. Spearman-rho test was used to checking the correlations. Multivariate analysis was done using multiple linear regression to define the independent factors that significantly affect the level of miRNA-146a$5 p$ expression in RA. A $p$-value of $<0.05$ was considered significant.

\section{Results}

The demographic data, expression of miRNA 1461-5p in patients and controls, clinical and laboratory data of the study group are listed in Tables $1 \& 2$. The level of expression of miRNA $146 a-5 p$ was compared between both the patients and the control group. The median expression level miRNA-146a-5p was significantly higher in the patients' group $(P=$ 0.036) (Table 1).

Table 1. Demographic Data and the Expression of miRNA 146a-5p in RA Patients and Controls.

\begin{tabular}{lccc}
\multicolumn{1}{c}{ Variable } & Cases N=60 & Controls N=45 & $P$ value \\
\hline Age $(\mathrm{y})$ (mean \pm SD) & $42 \pm 11.4$ & $39.5 \pm 10 . .3$ & NS \\
\hline Gender & $54(90 \%)$ & $37(82.2 \%)$ & NS \\
Females & $6(10 \%)$ & $8(17.8 \%)$ & \\
Males & & & \\
\hline miRNA 146a-5p & 0.958 & 0.242 & 0.036 \\
Median & $0.014-36.698$ & $0.074-16.309$ & \\
Min-Max & &
\end{tabular}

$P$ value $>0.05$ is not significant (NS) 
Table 2. Laboratory and Clinical Data of the Patients Group.

\begin{tabular}{|c|c|}
\hline Parameters & $\begin{array}{c}\text { RA patients } \\
\text { mean } \pm S D \text { (minimum and maximum) }\end{array}$ \\
\hline $\mathrm{ESR}(\mathrm{mm} / \mathrm{h})$ & $51.1 \pm 23.0(20-100)$ \\
\hline CRP (g/dl) & $22.5 \pm 20.0(3-86)$ \\
\hline $\mathrm{HB}(\mathrm{gm} / \mathrm{dl})$ & $11.9 \pm 1.3(9.8-15.3)$ \\
\hline WBCs (Cells $/ \mathrm{mm}^{3}$ ) & $7.74 \pm 2.8(2.4-13.2)$ \\
\hline $\mathrm{ALT}(\mathrm{mg} / \mathrm{dl})$ & $22.3 \pm 17.4(7-88)$ \\
\hline AST (mg/dl) & $25.7 \pm 16 . .7(7-82)$ \\
\hline S. Albumin (mg/dl) & $3.7 \pm 0.58(2.3-5.1)$ \\
\hline S. Creatinine (mg/dl) & $0.72 \pm 0.23(0.19-1.4)$ \\
\hline RF Positive (n/\%) & $34(76 \%)$ \\
\hline Anti-CCP (n/\%) & $32(53.3 \%)$ \\
\hline DAS28(ESR) & $5.27 \pm(2.95-7.41)$ \\
\hline $\mathrm{MHAQ}$ & $0.57 \pm 0.47(0-2.25)$ \\
\hline VAS & $51.7 \pm 19.5(10-80)$ \\
\hline Sharp Score & $126 \pm 141.3(4-420)$ \\
\hline Medications Steroids (n/\%) & $60(100 \%)$ \\
\hline Methotrexate only (n/\%) & $26(43.3 \%)$ \\
\hline Antimalarials only (n\%) & $4(6.7 \%)$ \\
\hline Leflunomide only (n\%) & $6(10 \%)$ \\
\hline Methotrexate and Antimalarial drugs (n/\%) & $10(16.7 \%)$ \\
\hline Methotrexate and Leflunomide (n/\%) & $2(3.3 \%)$ \\
\hline Leflunomide and Antimalarial drugs (n/\%) & $10(16.7 \%)$ \\
\hline Methotrexate, Leflunomide and Antimalarial drugs (n/\%) & $2(3.3 \%)$ \\
\hline
\end{tabular}


Table 3. Comparison of MiRNA146a expression levels and DMARDs therapy.

\begin{tabular}{|c|c|c|c|c|}
\hline Variable & No (\%) & Mean $\pm S D$ & Median & $P$ value \\
\hline \multicolumn{5}{|c|}{ MIRNA -146 a-5p: } \\
\hline \multicolumn{5}{|c|}{ Methotrexate } \\
\hline Yes & $20(33.3 \%)$ & $2.711 \pm 5.13$ & 0.565 & \multirow{2}{*}{ NS } \\
\hline NO & $40(66.7 \%)$ & $3.211 \pm 8.56$ & 0.153 & \\
\hline \multicolumn{5}{|l|}{ Leflunomide } \\
\hline Yes & $40(66.7 \%)$ & $3.30 \pm 8.55$ & 0.13 & \multirow{2}{*}{ NS } \\
\hline NO & $20(33.3 \%)$ & $2.51 \pm 5.14$ & 0.56 & \\
\hline \multicolumn{5}{|l|}{ Anti-Malarial } \\
\hline Yes & $34(56.7 \%)$ & $4.93 \pm 9.63$ & 0.91 & \multirow{2}{*}{0.016} \\
\hline NO & $26(43.3 \%)$ & $0.58 \pm 0.97$ & 0.12 & \\
\hline
\end{tabular}

$P$ value $>0.05$ is not significant (NS).

Comparison of miRNA 146a-5p expression level in RA patients was done in relation the disease activity scoring system DAS 28 , patients were divided into 2 groups according to DAS 28 . The first group included the patients with low and moderate score (Score $<3.2$ to 5.1) and the second group comprised patients with high DAS-28 $(>5.1)$, the difference between the 2 groups did not reveal a significant difference (mean: 1.95 and 3.87, respectively, $\mathrm{p}=0.06$ ). Similarly, no significant difference was detected between miRNA level and positivity of RF or anti-CCP $(P>0.05)$.

The miRNA-146a-5p expression level was significantly different between the patients group whose treatment protocol included antimalarial drugs in comparison to the other 2 groups in which antimalarials were not included in their treatment protocol (Table 4).

A multivariate linear regression analysis was used to detect the independent factors that significantly affect miRNA-146a-5p expression level-in RA patients. The results revealed that a high VAS is associated with a significant rise in miRNA-146a-5p expression level in RA patients ( $P=0.015)$ while the presence of antimalarial drugs in the patient' treatment protocol was significantly associated with a lower miRNA146a-5p expression level in RA patients ( $P$ $=0.021$ ) Table (4).

Table 4. Multivariate linear regression analysis for ESR, VAS and Anti-malarial drugs as independent factors affecting miRNA-146a-5p expression level.

\begin{tabular}{lccc}
\hline Variables & $\begin{array}{c}\text { Standardized } \\
\text { coefficients }\end{array}$ & $\mathrm{t}$ & $P$ value \\
\hline ESR & Beta & & $\mathrm{NS}$ \\
\hline VAS & 0.098 & 0.683 & 0.015 \\
\hline Anti-malarial Drugs & 0.302 & 2.509 & 0.021 \\
\hline
\end{tabular}

$P$ value $>0.05$ is not significant (NS). Note: miRNA-146a- $5 p$ is the dependent variable 
A significant correlation was detected between ESR and miRNA-146a-5p expression level ( $r=0.257, P=0.047$, respectively) (Figure 1 ). No correlation was detected between CRP and miRNA-146a-5 $p$ expression level $(r=0.087, P$ $=0.50)$.

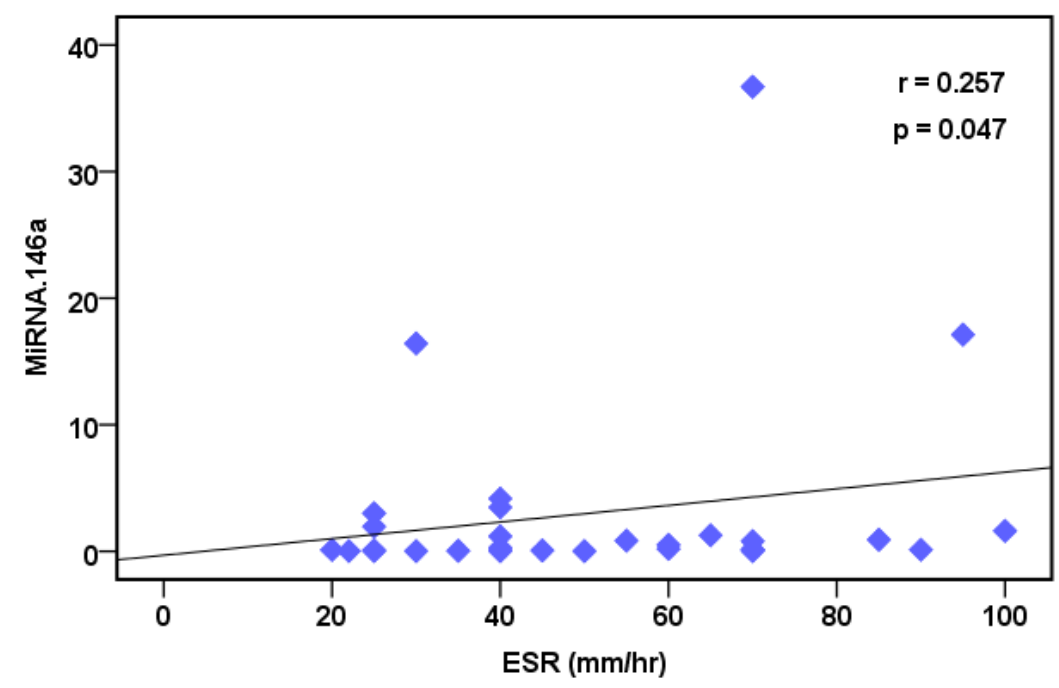

Figure 1. Correlation between miRNA-146a-5p expression level and ESR in RA Patients

On correlating miRNA-146a-5p expression level with disease activity and radiological severity, as represented by DAS28 and by Sharp score, respectively, a non- significant positive correlation was detected $(P=0.069$ and $P=0.14$, respectively). A significant positive correlation was detected between miRNA and both VAS as a measure of pain severity and MHAQ which is a predictor of long-term disease outcome ( $P=0.047$ and $P=0.050$, respectively) (Figures 2 \& 3).

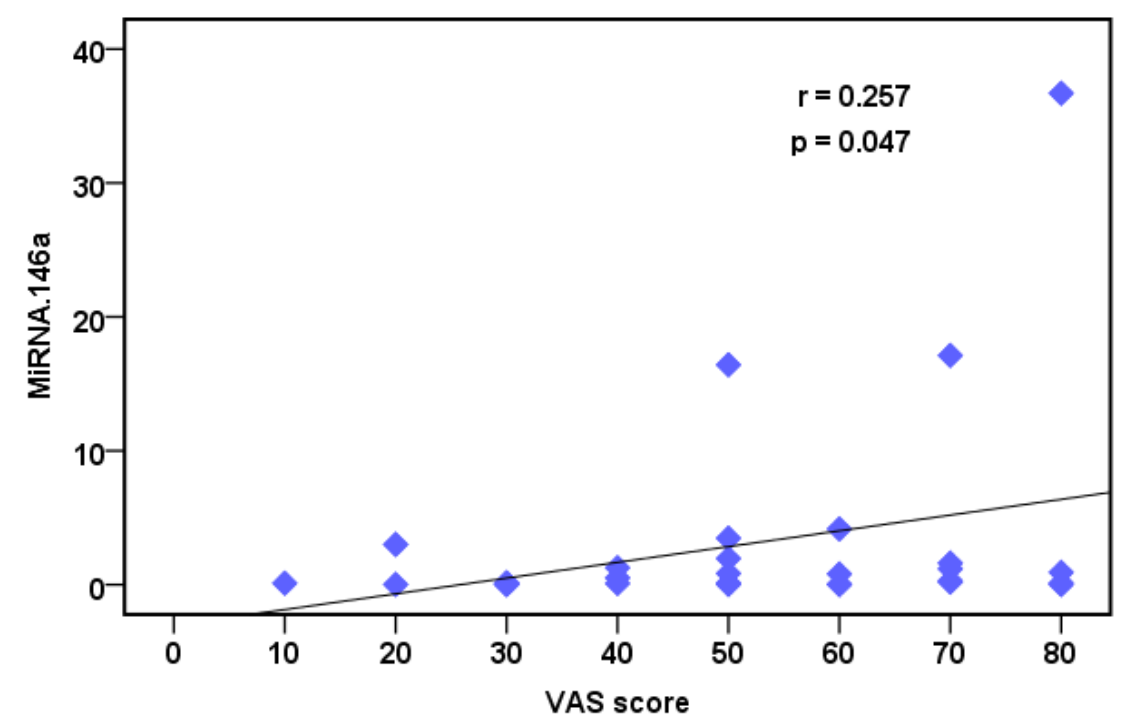

Figure 2. Correlation between VAS and miRNA-146a-5p expression level in RA patients. 


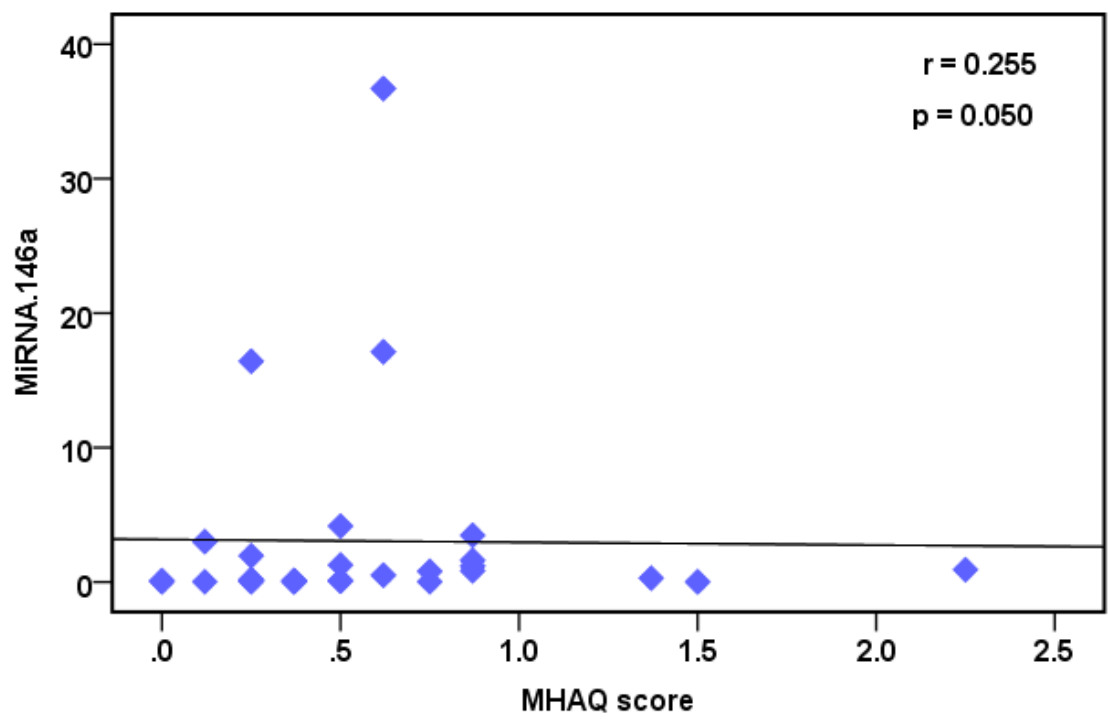

Figure 3. Correlation between MHAQ and miRNA-146a-5p expression level in RA patients

\section{Discussion}

In the current case control study, the expression of miRNA-146a-5p was evaluated in RA patients. The results showed that miRNA-146a-5p is overexpressed in the serum of RA patients $(P=0.036)$. These results are in agreement with the findings of Sun et al., 2018 and Ormseth et al., 2018, which disclosed that the mean expression level of miRNA-146a-5p was higher in RA patients compared to study controls. In addition, Pauley et al., 2008 and Abou-zeid et al., 2011, reported that miRNA-146a-5p expression level was increased in PBMCs of RA patients and in patients with other autoimmune diseases, in comparison to the study controls. ${ }^{5,15,16,17}$

Such results, of the present study and matched findings of other studies, might be attributed to several factors: (1) miRNA 146a-5p acts on II-17 causing the potentiation of $\mathrm{T}$ cell production and hence, the induction of the inflammatory state in RA; (2) miRNA 146a-5p might be implicated in the expression of the tumor necrosis factor (TNF) receptor associated factor 6 (TRAF6) and II-1 receptor associated kinase (IRAK-1) which leads to prolongation of TNF- $\alpha$ production ending in an exaggerated immune inflammatory response. MiRNA-146a$5 p$ expression might result in stimulating $\mathrm{TH} 1$ cells to produce IL12, IL-2 and IFN- $\gamma$ and hence accentuates the inflammatory response in RA. On the other hand, Wang et al., 2012 detected that circulating levels of miRNA-146a-5p were significantly down regulated in PBMCS in RA patients. $^{18,19}$

Contradictory outcomes were perceived as regards the relation between the expression level of miRNA 146a-5p and the disease activity as represented by DAS-28. In the current study, the mean expression level of miRNA 146a-5p was higher in the patient's group with high disease activity compared to those with low and moderate disease activity, however the difference did not reach statistical significance $(P=0.060)$. Similarly, Li et al., 2010 indicated that miRNA 146a-5p expression level was not associated with disease activity indices such as DAS28. $^{20}$ Nevertheless, Pauley et al., 2008, Abou-zeid et al., 2012 and Nakasa et al., 2011, detected the presence of an association between miRNA 146a-5p expression level and disease activity. ${ }^{16,19,20}$

In the present study, a significant correlation was detected between miRNA 146a-5p expression level and ESR which is one of the independent markers of disease activity $(P=0.047)$. This observation agreed with the results reported by Sun et al., 2018, and Nakasa et al., 2011. Thus, suggesting that the increase of miRNA 146a-5p expression may be closely 
associated with RA activity. ${ }^{5,21}$ Further on, in the present study, the level of miRNA 146a-5p was significantly correlated with VAS $(P=0.047)$. Similarly, Salim et al., $2017^{22}$ and Li et al., 2010 reported a positive correlation between miRNA $146 a-5 p$ expression levels and VAS values (as measure of the degree of pain which may indicate the degree of disease activity and severity).${ }^{20} \mathrm{~A}$ possible explanation for this is that miRNA 146a-5p affects the disease activity of RA through the mediation of various inflammatory and immune related genes and signaling pathways. MiRNA 146a-5p targets NF$\kappa \beta$ pathway prompting the excretion of several inflammatory cytokines including TNF- $\alpha$, IL-17 andIL-1 $\beta .^{23,24}$ On the other hand, Li et al., 2010 reported that they did not find a direct correlation between miRNA-146a expression level and disease activity. ${ }^{20}$

In the present work, vdHSS, a disease severity marker was not associated with MiRNA$146 a-5 p$ expression level. Although the results indicated the presence of a positive correlation; however, it did not reach a significant level $(P=0.147)$. On the contrary, Ayeldeen et al., 2018 indicated that the degree of joint damage was significantly associated with miRNA-146a$5 p$ expression level. ${ }^{25}$

As regards the medications of our RA patients, there was no significant difference in miRNA-146a-5p expression level in patients received Methotrexate or Leflunomide compared with patients who did not receive these medications $(P=0.347$ and $P=0.530$, respectively). Such findings agreed with those of Ayeldeen et al., 2018. However, Ayeldeen et al., 2018 did not detect a significant difference between miRNA-146a-5p expression level in RA patients on antimalarial therapy compared those who were not. ${ }^{25}$ On the other hand, there was a significant difference in the level of miRNA-146a-5p expression in RA patients received anti-malarial drugs compared to those who were treated with antimalarial drugs $(P=0.016)$.

The multivariate linear regression analysis revealed that antimalarial drugs might be one of the independent factors affecting the level of expression of miRNA-146a-5p $(P=0.021)$. The effect of antimalarial drugs on RA patients could be explained based on that one of the proposed mechanisms of action of anti-malarial drugs is to block T-cell responses through reducing proinflammatory cytokine production, including INF- $\gamma$, TNF, IL-1 and IL- $6^{26,27}$ and as miRNA-146a$5 p$ expression is increased by proinflammatory cytokines and is considered a modulator of differentiation and function of cells of the innate and adaptive immunity. ${ }^{22}$ Thus, taken together, antimalarial drugs might influence the reduction of miRNA 146a-5p expression level through reducing the production of proinflammatory cytokines as miRNA 146a-5p expression dependent on the production of proinflammatory cytokines. ${ }^{28}$

In conclusion, data of this study suggested that a possible association might be present between miRNA 146a-5p expression level and the incidence of RA. Treatment of RA patients with antimalarial drugs may result in lowered expression level of miRNA 146a-5p.

\section{Acknowledgment}

The authors of the study would like to thank all the participants in the study (patients and controls) for their participation, and nurses and technicians of our hospital for their efforts.

\section{Author Contributions}

RAE and RAB; conceptualization, sample collection and performed the laboratory work. SMF and EAA; examined the patients and performed the statistical results. All authors contributed in writing and reviewing the paper.

\section{Declaration of Conflicting Interests}

The author(s) declared no potential conflicts of interest with respect to the research, authorship, and/or publication of this article.

\section{Funding}

The author(s) denies receipt of any financial support for the research, authorship, and/or publication of this article.

\section{Ethical approval}

The study protocol was reviewed and approved by the Ethical Committee of the Faculty of Medicine, 
Beni-Suef University of Medicine (Approval No.: FMBSUREC/03092019).

\section{Informed consent}

An informed consent was obtained from each participant in the study.

\section{References}

1. Testa D, Calvacchi S, Petrelli F, et al. (2021). One year in review: Pathogenesis of rheumatoid arthritis. Clin Exep Rheumatol; 39 (3):445-452.

2. Chatzikyriakidou A, Voulgari P, Georgiou I, et al. (2012) "miRNAs and related polymorphisms in rheumatoid arthritis susceptibility," Autoimmunity Reviews, 11(9); 636-641.

3. Moran-Moguel M, Rio S, Mayorquin-Galvan E, et al. (2018). Rheumatoid Arthritis and miRNAs: A Critical Review through a Functional View. https://doi.org/10.1155/2018/2474529.

4. Banfai B, Jia H, Khatun J (2012). "Long noncoding RNAs are rarely translated in two human cell lines," Genome Research, 22(9); 1646-1657.

5. Sun G, Li J, Zhao H, et al. (2018). miR-146a and miR-155 as potential biomarkers for rheumatoid arthritis and disease activity. Int J Clin Exp Med; 11(9):9536-9543.

6. Taganov K, Boldin M, Chang K et al. (2006). NF-KBdependent induction of microRNA miR-146, an inhibitor targeted to signaling proteins of innate immune responses. Proc Natl Acad Sci USA 103(33): 12481-2486.37.

7. Furer V, Greenberg JD, Attur M, et al. (2010). The role of microRNA in rheumatoid arthritis and other autoimmune diseases. Clin Immunol; 136:1-15.

8. Elsayed $H$, Khater $W$, Ibrahim A, et al. (2017). MicroRNA-146a expression as a potential biomarker for rheumatoid arthritis in Egypt. Egyptian Journal of Medical Human Genetics, 18(2): 173-179.

9. Churov A, Oleinik E and Knip M (2015). MicroRNAs in rheumatoid arthritis: altered expression and diagnostic potential. Autoimmun Rev; 14:1029.

10. Aletaha D, Neogi T, Silman A, et al. (2010). Rheumatoid arthritis classification criteria: an American College of Rheumatology/European League Against Rheumatism collaborative initiative. Arthritis Rheum. 62: 2569-2581.

11. Pincus T, Summey J, Soraci S, et al. (1983). Assessment of patient satisfaction in activities of daily living using a modified Stanford Health Assessment Questionnaire. Arthritis Rheum; 26:1346-53.
12. Mc Cormack H, Horne D and Sheather S. (1988). Clinical applications of visual analogue scales: a critical review. Psychol Med; 18: 1007-19.

13. van Gestel AM, CJ Haagsma CJ, van Riel PL. (1998). Validation of rheumatoid arthritis improvement criteria that include simplified joint counts. Arthritis Rheum Oct; 41(10):1845-50.

14. Van Der Heijde $D$, van Reil $P$, van Leeunwen $M$, et al. (1992). Prognostic factors for radiographic damage and physical disability in early rheumatoid arthritis. A prospective study of 147 patients. $\mathrm{Br} J$ Rheumatol; 31(8):519-25.

15. Ormseth M, Solus J, Vickers K, et al (2015). Utility of select plasma microRNA for disease and cardiovascular risk assessment in patients with rheumatoid arthritis. J Rheumatol; 42:1746-51.

16. Pauley K, Satoh M, Chan A, et al. (2008). Upregulated miR-146a expression in peripheral blood mononuclear cells from rheumatoid arthritis patients. Arthritis Res Ther; 10:R101.

17. Abou-Zeid A, Saad M and Soliman E. (2011). MicroRNA 146a expression in rheumatoid arthritis: association with tumor necrosis factor-alpha and disease activity. Genet Test Mol Biomarkers; 15:80712.

18. Liu Y, Han Y, Qu H, et al. (2019) Correlation of microRNA expression profile with clinical response to tumor necrosis factor inhibitor in treating rheumatoid arthritis patients: A prospective cohort study. Journal of Clinical Laboratory Analysis. 33(7). https://doi.org/10.1002/jcla.22953

19. Wang H, Peng W, Ouyang X, et al. (2012). Circulating microRNAs as candidate biomarkers in patients with Systemic Lupus Erythematosus. Transl Res; 160(3):198-206.

20. Nakasa T, Shibuya H, Nagata $Y$, et al. (2011). The inhibitory effect of microRNA-146a expression on bone destruction in collagen-induced arthritis. Arthritis Rheum; 63:1582-1590.

21. Li J, Wan Y, Guo Q, et al. (2010). Altered microRNA expression profile with miR-146a upregulation in CD4+ $T$ cells from patients with rheumatoid arthritis. Arthritis Res Ther;12:R81

22. Salim R, Hassan T and Elnady B. (2017). MIR-146A and TLR4 gene expression in predicting rheumatoid arthritis disease. Egyptian Journal of Biochemistry and Molecular Biology 35:1-2.

23. Sonkoly E, Stahle M and Pivarcsi A (2008). MicroRNAs and immunity: novel players in the regulation of normal immune function and inflammation. Semin Cancer Biol., 18:131-140. doi: 10.1016/j.semcancer.2008.01.005. [PubMed] [CrossRef] [Google Scholar] 
24. Tang $Y$, Luo $X$, Cui $H$, et al (2009). MicroRNA-146A contributes to abnormal activation of the type I interferon pathway in human lupus by targeting the key signaling proteins. Arthritis Rheum. 60:10651075. doi:10.1002/art.24436. [PubMed] [CrossRef] [Google Scholar]

25. Ayeldeen G, Nassar Y, Ahmed H, et al. (2018). Possible use of miRNAs-146a and -499 expression and their polymorphisms as diagnostic markers for rheumatoid arthritis. Molecular and Cellular Biochemistry; 449(1-2):145-156.

26. Neto E, Kakihsi A, Pinheiro M, et al. (2020). Revisiting hydroxychloroquine and chloroquine for ptients with chronic immunity- mediated inflammatory rheumatic diseases. Advances in Rheumatology; 60(1):32. doi: 10.1186/s42358-02000134-8

27. Rusca N, Monticelli S (2011). MiR-146a in immunity and disease. Mol Biol Int 2011:43701 Article PubMed PubMed Central Google Scholar

28. Hurov AV, Oleinik EK and Knip M (2015). MicroRNAs in rheumatoid arthritis: altered expression and diagnostic potential. Autoimmun Rev 14:1029-1037 CAS Article PubMed Google Scholar 